\title{
Final Results of TACTICS: A Randomized, Prospective Trial Comparing Transarterial Chemoembolization Plus Sorafenib to Transarterial Chemoembolization Alone in Patients with Unresectable Hepatocellular Carcinoma
}

\begin{abstract}
Masatoshi Kudo ${ }^{a}$ Kazuomi Ueshima ${ }^{a}$ Masafumi lkeda ${ }^{b}$ Takuji Torimurac ${ }^{c}$ Nobukazu Tanabe ${ }^{d}$ Hiroshi Aikata ${ }^{e}$ Namiki Izumi ${ }^{f}$ Takahiro Yamasakig, h Shunsuke Nojiri ${ }^{i}$ Keisuke Hino ${ }^{j}$ Hidetaka Tsumurak $^{k}$ Teiji Kuzuyal Norio Isoda ${ }^{m}$ Michihisa Moriguchi ${ }^{n}$ Hajime Aino $^{\circ}$ Akio Ido $^{p}$ Naoto Kawabe ${ }^{l}$ Kazuhiko Nakao ${ }^{q}$ Yoshiyuki Wadar Sadahisa Ogasawara ${ }^{\mathrm{s}}$ Kenichi Yoshimura $^{\mathrm{t}}$

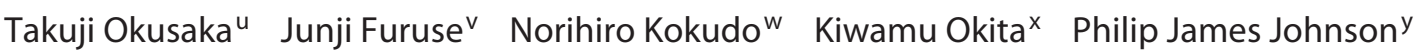
Yasuaki Arai ${ }^{z}$ on behalf of the TACTICS Study Group

aDepartment of Gastroenterology and Hepatology, Kindai University Faculty of Medicine, Osaka, Japan; bepartment of Hepatobiliary and Pancreatic Oncology, National Cancer Center Hospital East, Kashiwa, Japan; 'Division of Gastroenterology, Department of Medicine, Kurume University School of Medicine, Fukuoka, Japan; ${ }^{d}$ Department of Gastroenterology, National Hospital Organization Sendai Medical Center, Sendai, Japan; ${ }^{\text {DDepartment of }}$ Gastroenterology and Metabolism, Graduate School of Biomedical and Health Sciences, Hiroshima University, Hiroshima, Japan; fDepartment of Gastroenterology and Hepatology, Musashino Red Cross Hospital, Tokyo, Japan; gDepartment of Gastroenterology and Hepatology, Yamaguchi University Graduate School of Medicine, Yamaguchi, Japan; 'hepartment of Oncology and Laboratory, Yamaguchi University Graduate School of Medicine, Yamaguchi, Japan; 'Department of Gastroenterology and Metabolism, Nagoya City University Graduate School of Medical Science, Nagoya, Japan; 'Department of Hepatology and Pancreatology, Kawasaki Medical School, Okayama, Japan; kDepartment of Grastroenterological Oncology, Hyogo Cancer Center, Hyogo, Japan; 'Department of Gastroenterology and Hepatology, Fujita Health University School of Medicine, Toyoake, Japan; 'mivision of Gastroenterology, Department of Medicine, Jichi Medical University, Tochigi, Japan; ${ }^{n}$ Department of Molecular Gastroenterology and Hepatology, Kyoto Prefectural University of Medicine Graduate School of Medical Science, Kyoto, Japan; ${ }^{\circ}$ Division

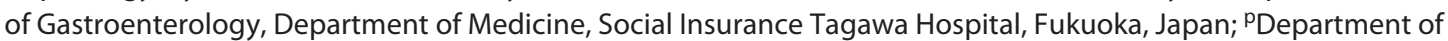
Gastroenterology and Hepatology, Kagoshima University Graduate School of Medical and Dental Sciences, Kagoshima, Japan; 'Department of Gastroenterology and Hepatology, Nagasaki University Graduate School of Biomedical Sciences, Nagasaki, Japan; 'Department of Hepato-Biliary-Pancreatic Surgery, National Hospital Organization Kyushu Medical Center, Fukuoka, Japan; ' Department of Gastroenterology, Graduate School of Medicine, Chiba University, Chiba, Japan; ${ }^{t}$ Medical Center for Translational and Clinical Research, Hiroshima University Hospital, Hiroshima, Japan; uDepartment of Hepatobiliary and Pancreatic Oncology, National Cancer Center Hospital, Tokyo, Japan; ${ }^{\vee}$ Department of Medical Oncology, Kyorin University Faculty of Medicine, Tokyo, Japan; wNational Center for Global Health and Medicine, Tokyo, Japan; ${ }^{X}$ Shunan Memorial Hospital, Yamaguchi, Japan; ${ }^{y}$ Department of Molecular and Clinical Medicine, University of Liverpool, Liverpool, UK; ${ }^{\mathrm{Z} D}$ Department of Diagnostic Radiology, National Cancer Center, Tokyo, Japan
\end{abstract}

Masatoshi Kudo and Kazuomi Ueshima contributed equally to this work.

Karger@karger.com www.karger.com/lic

Karger $\stackrel{\text { ' }}{5}$

GOPEN ACCESS
(C) 2022 The Author(s).

Published by S. Karger AG, Basel

This is an Open Access article licensed under the Creative Commons Attribution-NonCommercial-4.0 International License (CC BY-NC) (http://www.karger.com/Services/OpenAccessLicense), applicable to the online version of the article only. Usage and distribution for commercial purposes requires written permission.
Correspondence to:

Masatoshi Kudo, m-kudo@med.kindai.ac.jp 


\section{Keywords}

Hepatocellular carcinoma · Transarterial

chemoembolization · Sorafenib · Overall survival · Up-to-7

criteria

\section{Abstract}

Introduction: Several clinical trials comparing the efficacy and safety of transarterial chemoembolization (TACE) plus molecular-targeted agents versus TACE alone revealed no clinical benefits in progression-free survival (PFS) or overall survival (OS). Here, we report the final OS analysis from the TACTICS trial, which previously demonstrated significant improvement in PFS with TACE plus sorafenib in patients with unresectablehepatocellularcarcinoma(HCC)(NCT01217034). Methods: Patients with unresectable HCC were randomized to a TACE plus sorafenib group $(N=80)$ or a TACE alone group $(N=76)$. Patients in the combination treatment group received sorafenib $400 \mathrm{mg}$ once daily for 2-3 weeks before TACE, followed by 800 mg once daily during on-demand conventional TACE sessions until time to untreatable progression. In this trial, TACE-specific PFS was used. TACE-specific PFS is defined as the time from randomization to progressive disease (PD) or death from any cause, and PD was defined as untreatable progression, caused by the inability of a patient to further receive or benefit from TACE for reasons that include intrahepatic tumor progression ( $25 \%$ increase vs. baseline) according to response evaluation criteria in cancer of the liver, the detection of extrahepatic spread, vascular invasion, or transient deterioration of liver function to Child-Pugh C after TACE. Results: At the cut-off date of July 31, 2020, 131 OS events were observed. The median OS was 36.2 months with TACE plus sorafenib and 30.8 months with TACE alone (hazard ratio $[\mathrm{HR}]=0.861 ; 95 \%$ confidence interval $[\mathrm{Cl}]$, 0.607-1.223; $p=0.40, \Delta O S, 5.4$ months). The updated PFS was 22.8 months with TACE plus sorafenib and 13.5 months with TACE alone ( $\mathrm{HR}=0.661 ; 95 \% \mathrm{Cl}, 0.466-0.938 ; p=0.02)$. Posttrial treatments with active procedures/agents were received by $47(58.8 \%)$ patients in the TACE plus sorafenib group and $58(76.3 \%)$ in the TACE alone group $(p=0.01)$. In post hoc analysis, PFS and OS benefit were shown in HCC patients with tumor burden beyond up-to-7 criteria. Conclusions: In TACTICS trial, TACE plus sorafenib did not show significant OS benefit over TACE alone; however, clinical meaningful OS prolongation and significantly improved PFS was observed. Thus, the TACE plus sorafenib can be considered a choice of treatment in intermediate-stage HCC, especially in patients with high tumor burden. Trial Registration: NCT01217034.

(c) 2022 The Author(s).

Published by S. Karger AG, Basel

\section{Introduction}

Transarterial chemoembolization (TACE) is the most widely used locoregional therapy for intermediate-stage hepatocellular carcinoma (HCC) $[1,2]$ as recommended by the European Association for the Study of the Liver [3] and American Association for the Study of Liver Diseases (AASLD) [4] guidelines. However, TACE is not a curative treatment, and cancer recurrence eventually occurs in most cases [5]. Therefore, TACE is usually performed repeatedly, which leads to deterioration in liver function, often rendering the patient ineligible for systemic therapy. Additionally, TACE induces hypoxia and consequently elevated hypoxia-inducible factor 1 - $\alpha$ expression in tumors $[6,7]$. Elevated hypoxia-inducible factor 1- $\alpha$ levels cause upregulation of vascular endothelial growth factor (VEGF) and angiopoietin-2, leading to intrahepatic metastasis, metastasis to extrahepatic sites, and vascular invasion, thus deteriorating patient prognosis [7].

Antiangiogenic agents (VEGF inhibitors) reportedly normalize abnormal blood vessels, microvessel density, interstitial pressure, and vascular permeability in HCC [8]. These effects further improve drug delivery by TACE and might ultimately enhance its efficacy [9-11]. Additionally, delaying tumor progression by antiangiogenic agent without deteriorating liver function, potentially by prolonging the intervals between consecutive TACE sessions, may lead to more effective systemic therapies after progression on TACE.

Sorafenib is a multi-kinase inhibitor that suppresses the activities of the VEGF, RAF, and platelet-derived growth factor receptors. Therefore, in addition to its direct antitumor effect, sorafenib has an antiangiogenic inhibiting effect and is currently used as the first-line systemic therapy for advanced HCC to significantly prolong patient survival. Combining TACE with molecular-targeted agents that possess anti-VEGF activity, including sorafenib, may suppress hypoxia-inducible cytokines, such as VEGF and angiopoietin-2 following TACE. Various studies have focused on investigating the clinical benefits of TACE in combination with molecular-targeted agents that suppress angiogenesis. However, all the prospective randomized controlled trials performed to date considering progression-free survival (PFS) or overall survival (OS) [12-16] as endpoints have failed.

We conducted the TACTICS trial using TACE-specific PFS as the endpoint [17] rather than the modified Response Evaluation Criteria in Solid Tumors (RECIST). TACE-specific PFS is defined as the time from randomization to progressive disease (PD) or death from any 


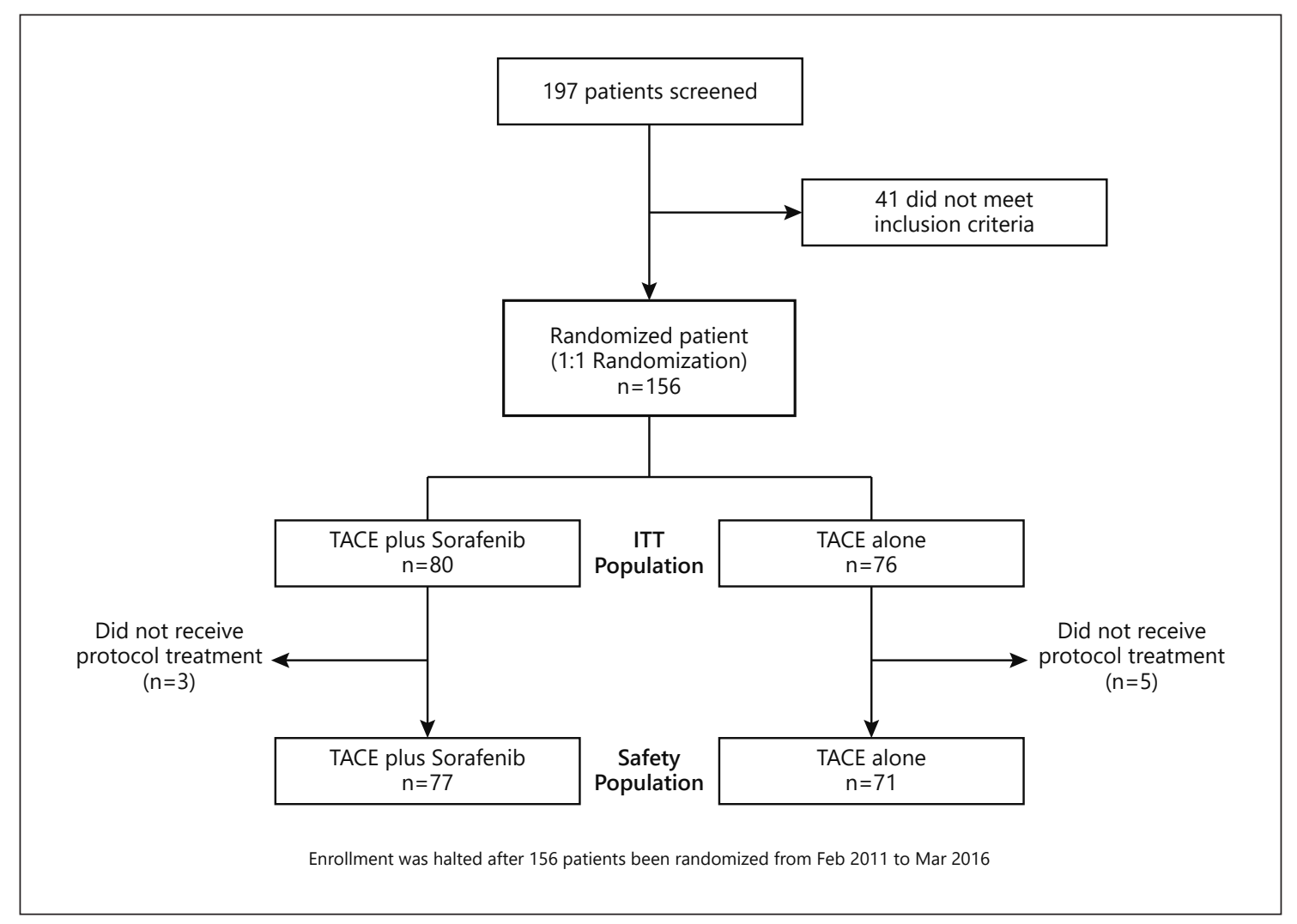

Fig. 1. Patient flowchart (CONSORT diagram).

cause, and PD was defined as untreatable (UnTACEable) progression, caused by the inability of a patient to further receive or benefit from TACE for reasons that include intrahepatic tumor progression ( $25 \%$ increase vs. baseline) according to response evaluation criteria in cancer of the liver (RECICL), the detection of extrahepatic spread (EHS), vascular invasion or transient deterioration of liver function to Child-Pugh C after TACE. This endpoint is in line with the criteria of progression under TACE treatment in routine clinical practice and TACE failure. This trial demonstrated significantly prolonged TACEspecific PFS [17]. Moreover, the treatment duration of the TACTICS trial was considerably longer than that of previous studies [12-16]. The SPACE and post-TACE studies showed that a prolonged duration of sorafenib treatment leads to clinical benefits, supporting that the prolonged treatment duration led to the success of the TACTICS trial.

Regrowth and appearance of new lesions naturally and frequently occur in patients with HCC treated by TACE. However, this progression alone does not necessarily indicate the failure of TACE or a need to switch to the next line of treatment in clinical practice. Therefore, because it would be inconsistent with routine clinical practice to stop TACE based on the determination of PD using the RECIST v1.1 or mRECIST, we used TACE-specific PFS, a composite endpoint unique to TACE. Although TACEspecific PFS was significantly prolonged in the primary analysis of the TACTICS trial [17], the number of protocol-specified death events was insufficient to evaluate the co-primary endpoint of OS. Thus, we here report the final results of the TACTICS trial in relation to OS, which was obtained after achieving the required number of events.

\section{Patients and Methods}

Design, Inclusion Criteria, and Ethics

This randomized, open-label, multicenter prospective trial was conducted at 33 centers across Japan between 2011 and 2020, which included patients with unresectable HCC diagnosed using tissue biopsy, cytology, or dynamic computed tomography (CT) and magnetic resonance imaging scans according to AASLD criteria. All patients included in the study were older than 20 years and had a life expectancy of 12 weeks or longer, with tumors localized in the liver without vascular invasion or extrahepatic metas- 
Table 1. Baseline demographic and clinical characteristics of patients enrolled in this study

\begin{tabular}{|c|c|c|}
\hline Characteristic & $\begin{array}{l}\text { TACE plus sorafenib } \\
(n=80)\end{array}$ & $\begin{array}{l}\text { TACE alone } \\
(n=76)\end{array}$ \\
\hline Age, median (range), years & $72.0(36-85)$ & $73.0(53-86)$ \\
\hline \multicolumn{3}{|l|}{ Sex } \\
\hline Male & $63(78.8)$ & $55(72.4)$ \\
\hline Female & $17(21.2)$ & $21(27.6)$ \\
\hline \multicolumn{3}{|l|}{ Performance status } \\
\hline 0 & $71(88.8)$ & $67(88.2)$ \\
\hline 1 & $9(11.3)$ & $9(11.8)$ \\
\hline \multicolumn{3}{|l|}{ Etiology } \\
\hline Hepatitis B & $10(12.5)$ & $2(2.6)$ \\
\hline Hepatitis C & $38(47.5)$ & $53(69.7)$ \\
\hline Non B non C & $32(40.0)$ & $21(27.6)$ \\
\hline \multicolumn{3}{|l|}{ Child-Pugh score } \\
\hline 5 & $64(80.0)$ & $54(71.1)$ \\
\hline 6 & $15(18.8)$ & $17(22.4)$ \\
\hline 7 & $1(1.3)$ & $5(5.6)$ \\
\hline \multicolumn{3}{|l|}{ AFP } \\
\hline$<200 \mathrm{ng} / \mathrm{mL}$ & $64(80.0)$ & $60(78.9)$ \\
\hline$\geq 200 \mathrm{ng} / \mathrm{mL}$ & $16(20.0)$ & $16(21.1)$ \\
\hline \multicolumn{3}{|l|}{ Tumor burden } \\
\hline Within Milan criteria & $28(35.0)$ & $35(46.1)$ \\
\hline Outside Milan criteria & $52(65.0)$ & $41(53.9)$ \\
\hline \multicolumn{3}{|l|}{ Up to 7 criteria } \\
\hline Within & $54(67.5)$ & $50(65.8)$ \\
\hline Outside & $26(32.5)$ & $26(34.2)$ \\
\hline \multicolumn{3}{|l|}{$\mathrm{BCLC}$ stage } \\
\hline$A^{*}$ & $27(33.8)^{*}$ & $33(43.4)^{*}$ \\
\hline B & $44(55.0)$ & $34(44.7)$ \\
\hline$C^{* *}$ & $9(11.3)$ & $9(11.8)$ \\
\hline \multicolumn{3}{|l|}{ Prior TACE } \\
\hline 0 & $45(56.3)$ & $48(63.2)$ \\
\hline $1-2$ & $35(43.8)$ & $28(36.8)$ \\
\hline
\end{tabular}

Results are reported as $n(\%)$, unless otherwise indicated. * Most of these nodules are single tumors larger than $5 \mathrm{~cm}$ in diameter. ** PS 1, no vascular invasion, no extrahepatic spread.

tasis. The patients had a maximum of 10 tumors, each with a maximum size of $10 \mathrm{~cm}$. Patients who had received $1-2$ prior TACE procedures were allowed to enroll in the study provided that the previous TACE was performed more than 6 months before the enrolment. The participants had an Eastern Cooperative Oncology Group performance status of zero or one, Child-Pugh score $\leq 7$, and well-preserved organ function. Patients with a history of systemic chemotherapy were excluded. Other exclusion criteria are described in detail in the previous manuscript describing the TACE-specific PFS results [17].

The study was conducted in accordance with the Declaration of Helsinki. The study was registered at ClinicalTrials.gov (NCT01217034) and was approved by the institutional review boards and/or independent Ethics Committees of the participating institutions. All patients provided written informed consent prior to enrolment.

TACE Plus Sorafenib in Unresectable HCC

\section{Randomization and Masking}

Patients were randomized 1:1 to treatment with either TACE plus sorafenib or TACE alone. Randomization was performed by a centralized data center using an interactive web response system involving a computer-generated sequence and electric data capture system software (Viedeoc, Uppsala, Sweden). Patient allocation factors were: (1) site, (2) meeting or not meeting Milan criteria (one lesion $\leq 5 \mathrm{~cm}$ or $\leq 3$ lesions of $\leq 3 \mathrm{~cm}$ each), and (3) number of prior TACE sessions (0 vs. 1-2 times).

\section{Treatment Protocol}

Patients randomized to the TACE plus sorafenib group were administered $400 \mathrm{mg}$ /day sorafenib, which is half of the recommended dose, $2-3$ weeks before the first TACE. The objective was to confirm tolerability to sorafenib for the management of adverse events (AEs) prior to TACE, as well as to normalize the tumor neovasculature for efficient TACE and suppress the post-TACE increase in hypoxia-inducing cytokines, such as VEGF and plateletderived growth factor. Sorafenib treatment was discontinued 2 days before the TACE session and resumed 3 days after the session.

In both groups, a lipidol plus epirubicin or miriplatin suspension was administered during TACE, followed by administration of an embolic agent (Gelpart) to embolize the tumor-feeding artery. The anticancer agent (epirubicin or miriplatin) was selected at the discretion of the site and investigators; however, the same anticancer agent was used in subsequent repeated TACE sessions for each patient. CT scans were acquired 4 weeks after the TACE session to evaluate the tumor response to the first treatment. Repeat TACE was recommended if the viable lesions (sum of twodimensional measurements of $\leq 5$ intrahepatic lesions) exceeded $50 \%$ of the tumor volume at baseline before the first TACE session.

Patients in the TACE plus sorafenib group were administered $400 \mathrm{mg} /$ day of sorafenib 3 days after the TACE session. If this dose was sufficiently tolerated, the dose was increased to $800 \mathrm{mg}$ /day at the discretion of the investigator. The tumor response was evaluated by dynamic CT and magnetic resonance imaging every 8 weeks. Treatments were continued until unTACEable progression, progression to meet the TACE refractory criteria [18], intolerable toxicity, or withdrawal of informed consent.

\section{Study Endpoints}

The co-primary endpoint of this trial was TACE-specific PFS and OS. For multiplicity adjustments, an endpoint was tested using a gatekeeping strategy, such that OS was analyzed only if the effect for TACE-specific PFS was significant [19]. TACE-specific PFS was defined as the time from randomization to progression or death from any cause, and OS was defined as the time from randomization to death from any cause. In this trial, progression was defined as unTACEable progression, which is the inability of a patient to further receive or benefit from TACE for reasons that included intrahepatic tumor progression (25\% increase of viable area in the sum of the five largest intrahepatic lesions vs. baseline) according to RECICL [20], transient deterioration of liver function to Child-Pugh grade $\mathrm{C}$ immediately after TACE, the appearance of macrovascular invasion (MVI), or EHS. New intrahepatic lesions were not regarded as "progressive disease," as they indicate the natural tumor biology of HCC and do not imply TACE failure or moving to the next line of treatment.

Neither RECIST 1.1 nor modified RECIST were used in this trial. Rather, tumor response was evaluated using RECICL [20]. A 


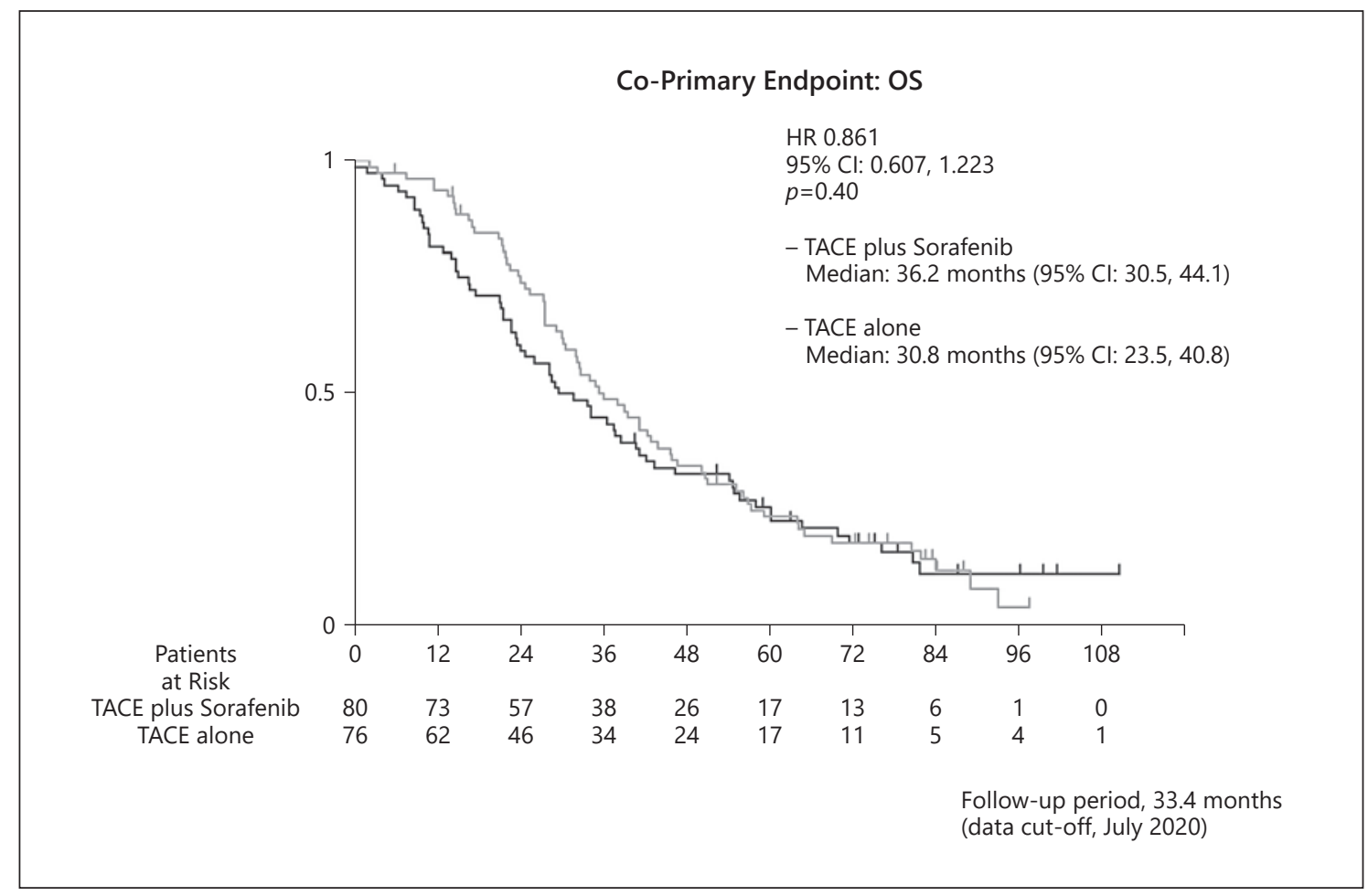

Fig. 2. Kaplan-Meier curves of the co-primary endpoint of OS in the TACTICS trial. The median observation period was 33.4 months (range, $0.1-110.7$ ) with 131 OS events.

lipiodol retention area in the nodule at 1 month after TACE was regarded as necrosis [21]. Therefore, a complete tumor response to TACE was defined as complete lipiodol retention within the nodule, and a partial tumor response was defined as $50-100 \%$ lipiodol retention within the nodule throughout the trial. Progression also included the Japan Society for Hepatology criteria for TACE failure/refractoriness [18].

Other secondary endpoints included the objective response rate after the first session of TACE and safety. Prespecified analyses included TACE-specific PFS and OS in patients with a tumor burden beyond up-to-seven criteria [22]. Other exploratory analysis included time from randomization to MVI or EHS (diameter $\geq 10$ $\mathrm{mm}$ ) and time to stage progression, which was defined as the time to the appearance of MVI and/or EHS.

\section{Statistical Methods}

All statistical analyses included in the primary analysis [17] were performed using the data obtained by the cut-off date of November 2, 2017, at which time point 118 patients had experienced disease progression or death. In the final analysis, statistical analysis was performed using the data obtained by the cut-off date of July 31, 2020, at which point 131 death events were observed. Efficacy data were analyzed on an intention-to-treat basis. To adjust for multiple comparisons, hierarchical statistical testing was performed in a prespecified fixed order, first for TACE-specific PFS and then for OS. According to the study protocol, OS was formally compared only when the difference in TACE-specific PFS between groups was significant $(p<0.15)$. The primary endpoints were compared using stratified log-rank tests. The Kaplan-Meier method was used to estimate survival curves, from which medians were calculated. Hazard ratios (HRs) and 95\% confidence intervals (CIs) were estimated using Cox proportional hazards models. Safety analysis included all patients who received at least one dose of the assigned treatment, irrespective of eligibility or duration of treatment. All statistical analyses were performed using SAS, V.9.4. (SAS Institute, Cary, NC, USA). The trial was designed to include 125 TACE-specific PFS and OS events to achieve a power of $80 \%$ with an HR of 0.71 and a one-sided alpha of 0.15 [17].

\section{Patient and Public Involvement}

There was no patient or public involvement in this study.

\section{Results}

\section{Patient Characteristics}

A total of 197 patients were screened between February 2011 and March 2016; of these, 156 met the inclusion criteria and were enrolled and randomized. Forty-one patients did not meet the inclusion criteria because of the Child-Pugh score, number of prior TACE procedures, and laboratory test results (Fig. 1). Among the 156 enrolled patients, 60 (38\%) had tumors categorized as Barcelona Clinic Liver Cancer (BCLC) stage A; most patients 


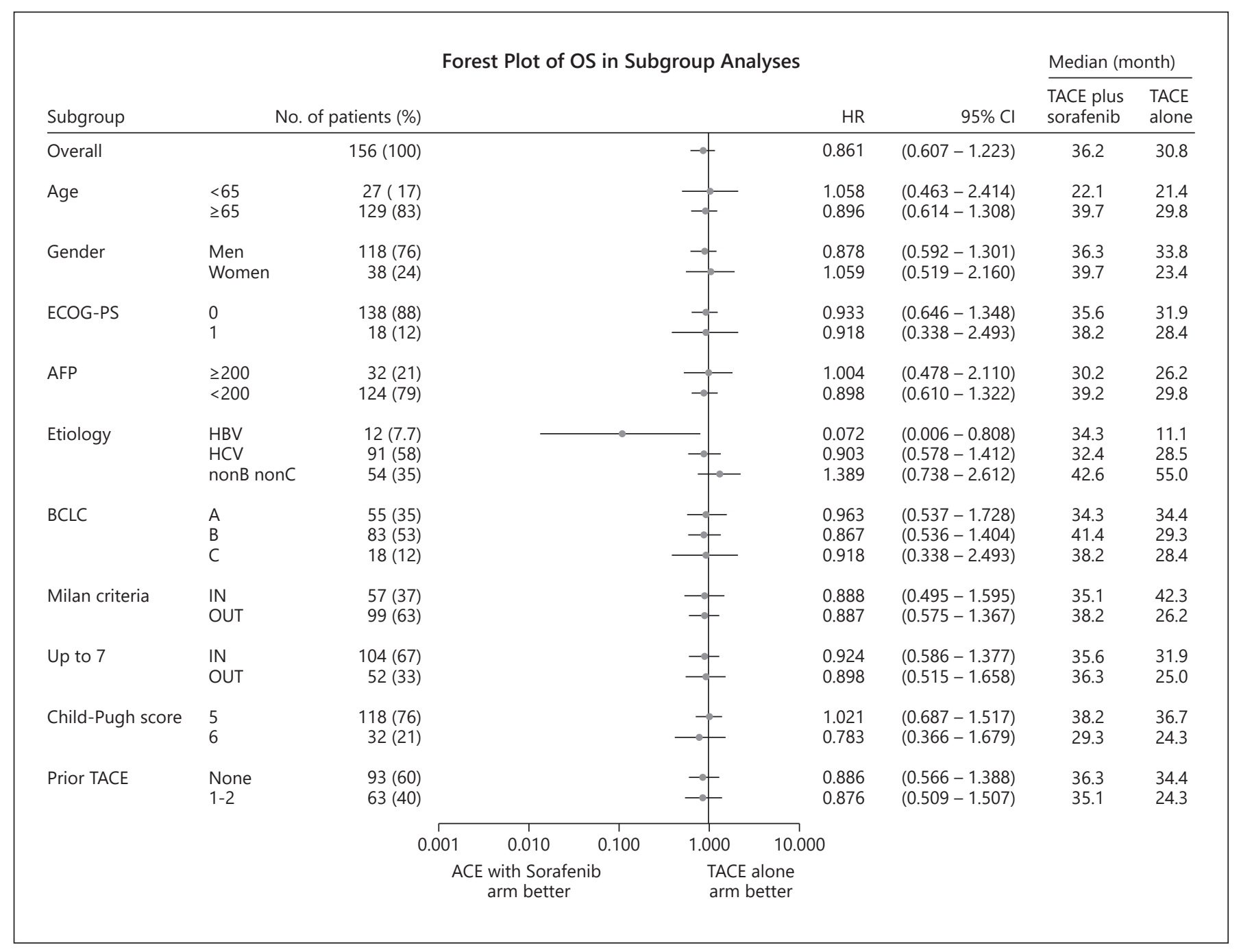

Fig. 3. Forest plot of OS in subgroup analysis.

had single large $(>5 \mathrm{~cm})$ unresectable tumor and were thus regarded as good candidates for TACE. Eighteen patients (12\%) had tumors categorized as BCLC stage C based on a performance status of 1 without EHS or MVI. The remaining 78 patients (50\%) had tumors categorized as BCLC stage B (Table 1). Overall, 80 patients were randomized to the TACE plus sorafenib group and 76 to the TACE alone group (Fig. 1). The baseline demographic and clinical characteristics were similar between the TACE plus sorafenib and TACE alone groups (online suppl. Table 1; for all online suppl. material, see www. karger.com/doi/10.1159/000522547). Additionally, the baseline demographic and clinical characteristics were similar in both arms for patients within and beyond the up-to-seven criteria (online suppl. Table 1).

TACE Plus Sorafenib in Unresectable HCC

\section{Doses of Study Drugs}

At a median follow-up of 33.4 months (range, 0.1-110.7 months), the median duration of sorafenib treatment in the TACE plus sorafenib group was 16.1 months (range, $0.2-78.2$ months). In 42 of 80 patients in the combination group, the sorafenib dose was increased to $800 \mathrm{mg}$ after the first TACE procedure; however, the dose was eventually reduced during treatment for all 42 patients because of either AEs or the physician's decision. The median actual daily dose of sorafenib was $355.2 \mathrm{mg}$ (range, 86.8-792.9 $\mathrm{mg}$ ), and the mean \pm SD actual daily dose of sorafenib was $353.6 \pm 172.0 \mathrm{mg}$. The median interval between TACE sessions was 23.2 weeks (range, 15.8-43.1 weeks) in the TACE plus sorafenib group and was 16.1 weeks (range, 10.1-32.1 weeks $)$ in the TACE alone group $(p=0.011)$. 

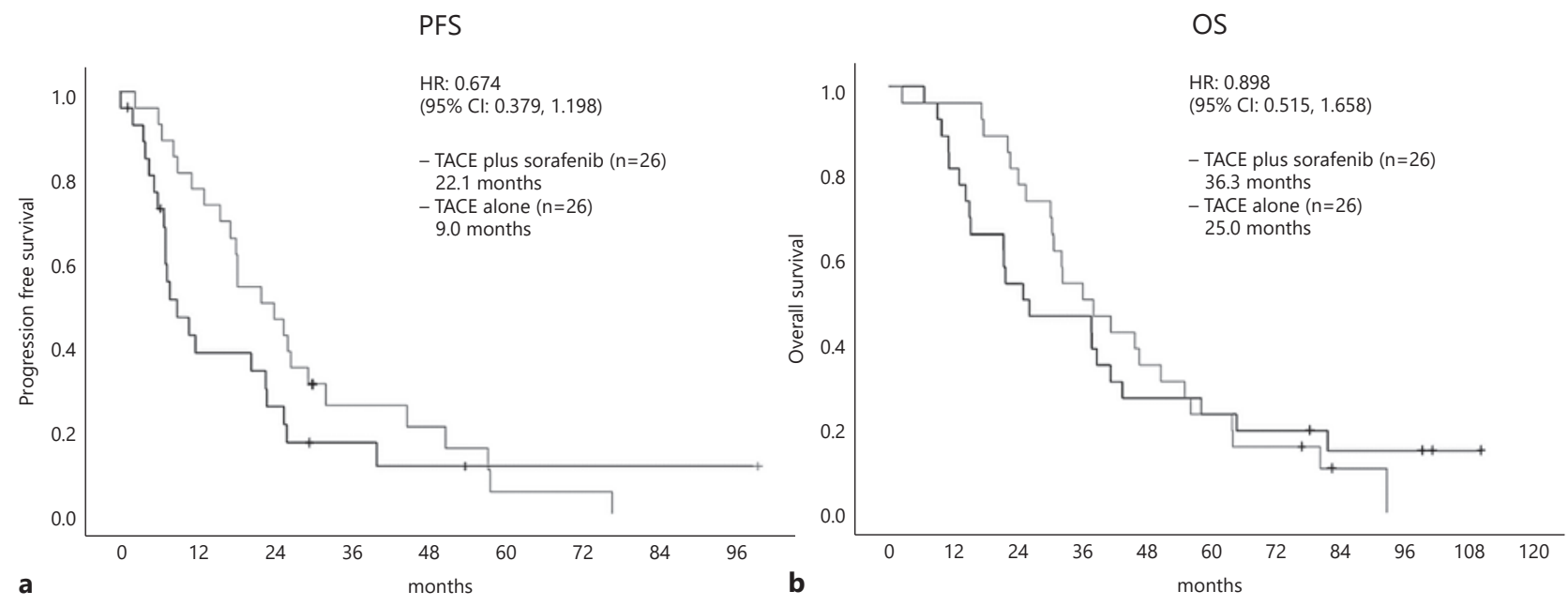

Within the Up-to-7 Criteria
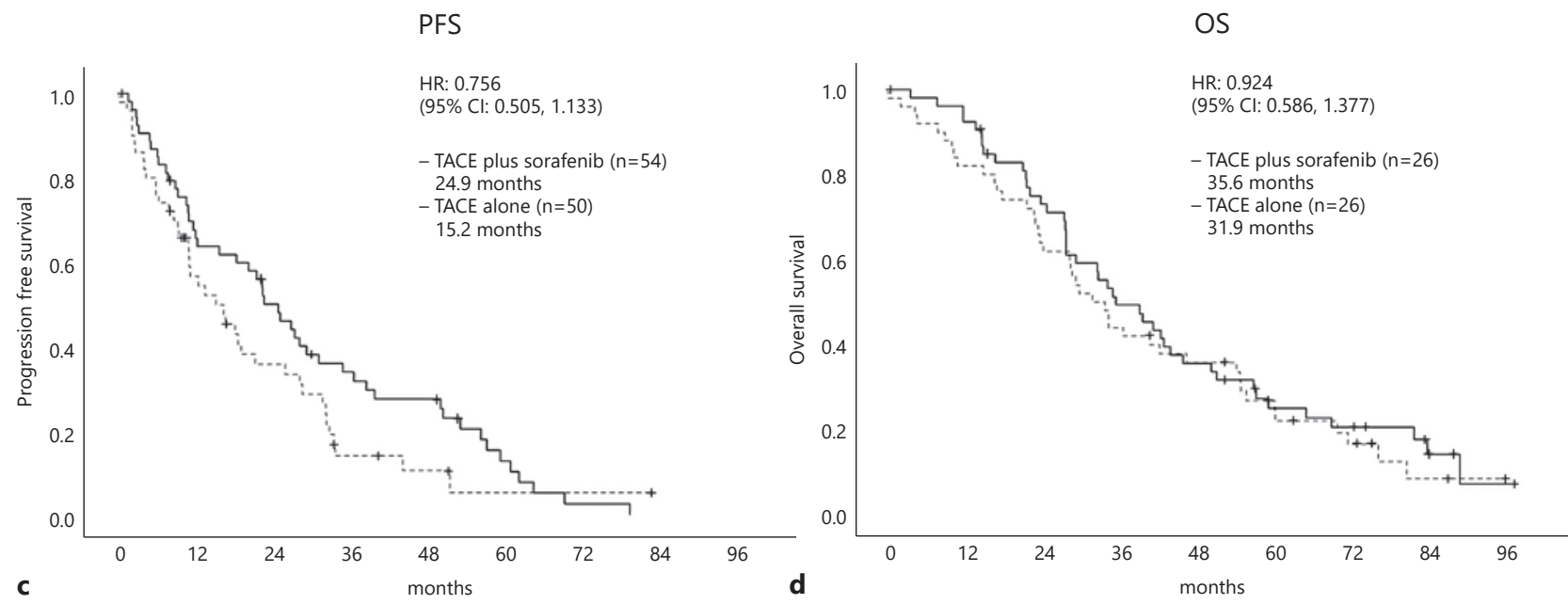

Fig. 4. TACE-specific PFS (a) and OS (b) in patients with tumor burden beyond the up-to-seven criteria. TACEspecific PFS (c) and OS (d) in patients with a tumor burden within the up-to-seven criteria.

\section{Efficacy Outcome}

The results of primary analysis of TACE-specific PFS, the co-primary endpoint, were previously published [17]. The data indicated that TACE-specific PFS was significantly longer in the TACE plus sorafenib group than in the TACE alone group (25.2 vs. 13.5 months; $\mathrm{HR}=0.59$; 95\% CI, 0.41-0.87; $p=0.006$ ) [17].
Because the PFS endpoint was positive, we analyzed OS, the other co-primary endpoint, in a predetermined manner in this study. The median OS in the TACE plus sorafenib group was 36.2 months (95\% CI, 30.5-44.1), whereas that in the TACE monotherapy group was 30.8 months (95\% CI, 23.5-40.8). Thus, TACE plus sorafenib did not provide a significantly greater survival benefit 


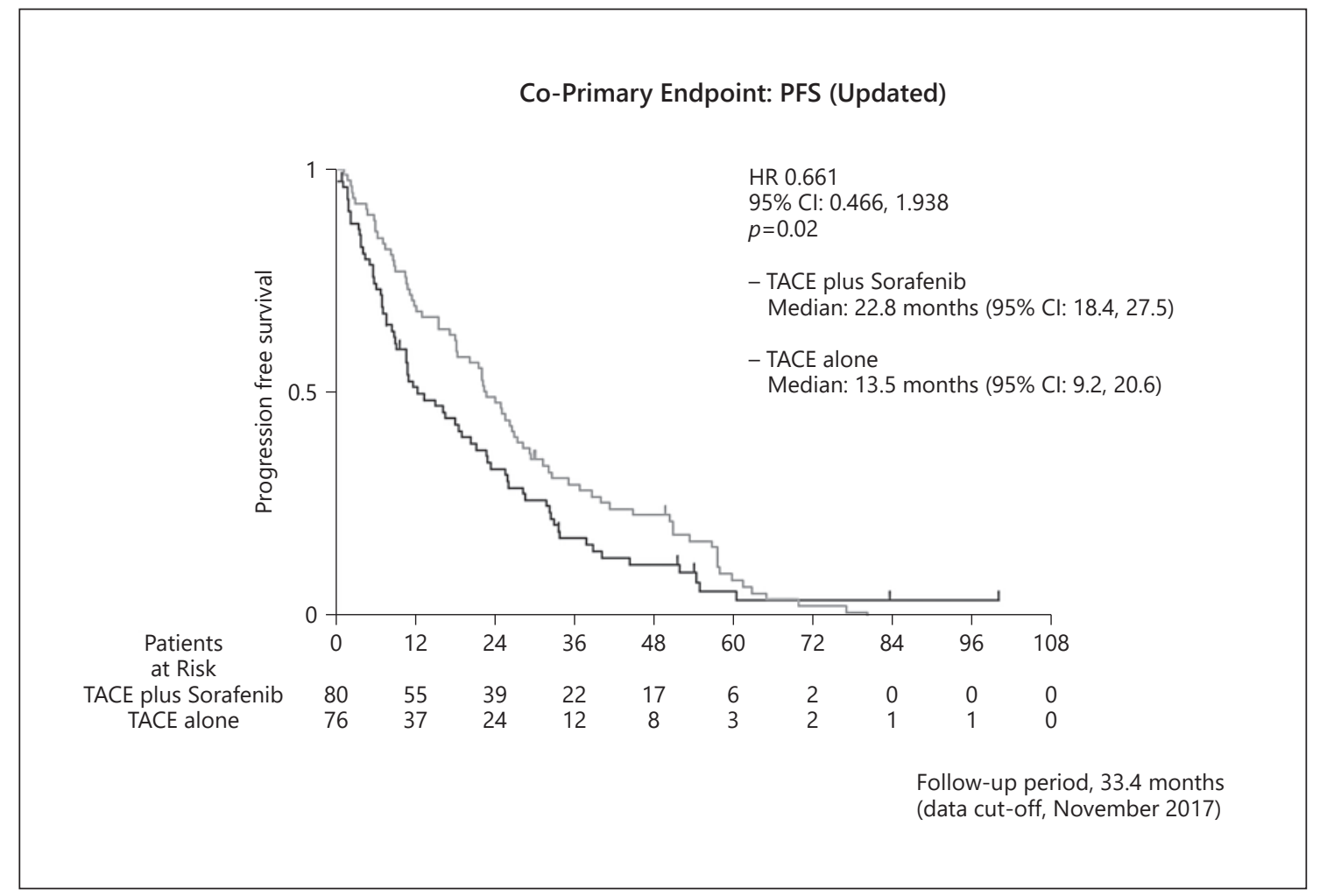

Fig. 5. Kaplan-Meier curves of the co-primary endpoint of TACE-specific PFS updated as of the data cut-off date of July 31, 2020. Consistent with the primary analysis, significantly longer TACE-specific PFS was observed in the TACE plus sorafenib arm than in the TACE alone arm.

than TACE monotherapy $(\mathrm{HR}=0.861 ; 95 \% \mathrm{CI}, 0.607-$ 1.223; $p=0.40$ ) (Fig. 2). Subgroup analysis showed that OS was better in the TACE plus sorafenib group than in the TACE monotherapy group when accounting for most factors (Fig. 3). Prespecified analysis showed that in patients outside the up-to-seven criteria, the median OS was 25.0 months in the TACE alone group and was 36.3 months in the TACE plus sorafenib group, representing numerically improvement of 11.3 months (Fig. 3, 4). Even among those within the up-to-seven criteria, the median OS in the TACE alone and TACE plus sorafenib groups was 31.9 and 35.6 months, respectively, representing numerically improvement of 3.7 months (Fig. 3, 4).

An updated repeat analysis of TACE-specific PFS, the co-primary endpoint, showed that the median TACEspecific PFS was 22.8 months (95\% CI, 18.4-27.5) in the TACE plus sorafenib group and was 13.5 months (95\% CI, 9.2-20.6) in the TACE monotherapy group. These data are consistent with those of the primary analysis (HR $=0.661 ; 95 \%$ CI, 0.466-0.938; $p=0.02$ ) (Fig. 5). The forest plot also showed that the TACE-specific PFS of the TACE plus sorafenib group was better than that of the TACE

TACE Plus Sorafenib in Unresectable HCC monotherapy group with respect to almost all factors. Prespecified analysis showed that the median TACE-specific PFS of patients outside the up-to-seven criteria was 9.0 months in the TACE monotherapy group, which was numerically prolonged to 22.1 months in the TACE plus sorafenib group $(\mathrm{HR}=0.674 ; 95 \% \mathrm{CI}, 0.379-1.198)$ (Fig. 4, 6). Similarly, the median TACE-specific PFS for patients within the up-to-seven criteria was numerically prolonged from 15.2 months in the TACE alone group to 24.9 months in the TACE plus sorafenib group (HR = 0.756; 95\% CI, 0.505-1.133) (Fig. 4, 6). The additive effects of sorafenib prolonged TACE-specific PFS by 9.7 months in the population within the up-to-seven criteria and by 13.1 months in the population outside the up-toseven criteria (Fig. 4, 6). The survival rates and TACEspecific PFS at each landmark point were better in the TACE plus sorafenib group than in the TACE alone group (online suppl. Table 2, 3).

\section{Post-Trial Anticancer Treatment}

Post-trial anticancer treatments were administered to 58 patients $(76.3 \%)$ in the TACE monotherapy group and 
Forest Plot of PFS in Subgroup Analyses (updated)

\begin{tabular}{|c|c|c|c|c|c|c|c|c|c|}
\hline \multirow[b]{2}{*}{ Subgroup } & \multicolumn{7}{|c|}{ Forest Plot of PFS in Subgroup Analyses (updated) } & \multicolumn{2}{|c|}{ Median PFS (month) } \\
\hline & \multicolumn{2}{|c|}{ No. of patients (\%) } & & & & $\mathrm{HR}$ & $95 \% \mathrm{Cl}$ & \multirow{2}{*}{$\begin{array}{c}\text { TACE plus } \\
\text { sorafenib } \\
22.8\end{array}$} & \multirow{2}{*}{$\begin{array}{r}\text { TACE } \\
\text { alone }\end{array}$} \\
\hline Overall & & $156(100)$ & & $\rightarrow$ & & 0.661 & $(0.466-0.938)$ & & \\
\hline Age & $\begin{array}{l}<65 \\
\geq 65\end{array}$ & $\begin{array}{l}27(17) \\
129(8)\end{array}$ & & $\rightarrow-$ & - & $\begin{array}{l}0.652 \\
0.727\end{array}$ & $\begin{array}{l}(0.272-1.564) \\
(0.506-1.044)\end{array}$ & $\begin{array}{l}13.2 \\
25.2\end{array}$ & $\begin{array}{r}6.9 \\
13.4\end{array}$ \\
\hline Gender & $\begin{array}{l}\text { Men } \\
\text { Women }\end{array}$ & $\begin{array}{r}118(76) \\
38(24)\end{array}$ & & $\rightarrow-$ & & $\begin{array}{l}0.760 \\
0.710\end{array}$ & $\begin{array}{l}(0.520-1.112) \\
(0.363-1.390)\end{array}$ & $\begin{array}{l}22.8 \\
24.9\end{array}$ & $\begin{array}{l}13.5 \\
11.0\end{array}$ \\
\hline ECOG-PS & $\begin{array}{l}0 \\
1\end{array}$ & $\begin{array}{r}138(88) \\
18(12)\end{array}$ & & $\rightarrow$ & & $\begin{array}{l}0.782 \\
0.480\end{array}$ & $\begin{array}{l}(0.549-1.112) \\
(0.179-1.289)\end{array}$ & $\begin{array}{l}22.5 \\
29.4\end{array}$ & $\begin{array}{l}11.9 \\
13.5\end{array}$ \\
\hline AFP & $\begin{array}{l}\geq 200 \\
<200\end{array}$ & $\begin{array}{r}32(21) \\
124(79)\end{array}$ & & - & - & $\begin{array}{l}1.016 \\
0.671\end{array}$ & $\begin{array}{l}(0.483-2.138) \\
(0.464-0.971)\end{array}$ & $\begin{array}{l}13.2 \\
25.2\end{array}$ & $\begin{array}{l}10.8 \\
15.2\end{array}$ \\
\hline Etiology & $\begin{array}{l}\text { HBV } \\
\text { HCV } \\
\text { nonB nonC }\end{array}$ & $\begin{array}{l}12(7.7) \\
91(58) \\
54(35)\end{array}$ & & - & - & $\begin{array}{l}0.258 \\
0.817 \\
0.808\end{array}$ & $\begin{array}{l}(0.038-1.764) \\
(0.532-1.254) \\
(0.444-1.468)\end{array}$ & $\begin{array}{l}13.2 \\
21.6 \\
29.4\end{array}$ & $\begin{array}{r}6.9 \\
12.4 \\
18.6\end{array}$ \\
\hline BCLC & $\begin{array}{l}\text { A } \\
\text { B } \\
\text { C }\end{array}$ & $\begin{array}{r}51(33) \\
103(66) \\
18(12)\end{array}$ & & 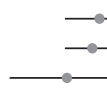 & & $\begin{array}{l}0.827 \\
0.727 \\
0.480\end{array}$ & $\begin{array}{l}(0.475-1.437) \\
(0.458-1.155) \\
(0.179-1.289)\end{array}$ & $\begin{array}{l}25.2 \\
22.1 \\
29.3\end{array}$ & $\begin{array}{l}16.3 \\
11.1 \\
13.5\end{array}$ \\
\hline Milan criteria & $\begin{array}{l}\text { IN } \\
\text { OUT }\end{array}$ & $\begin{array}{l}57(37) \\
99(63)\end{array}$ & & 一- & & $\begin{array}{l}0.807 \\
0.683\end{array}$ & $\begin{array}{l}(0.469-1.388) \\
(0.449-1.039)\end{array}$ & $\begin{array}{l}26.9 \\
22.5\end{array}$ & $\begin{array}{l}18.6 \\
10.9\end{array}$ \\
\hline Up to 7 & $\begin{array}{l}\text { IN } \\
\text { OUT }\end{array}$ & $\begin{array}{r}104(67) \\
52(33)\end{array}$ & & 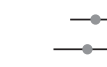 & & $\begin{array}{l}0.756 \\
0.674\end{array}$ & $\begin{array}{r}(0.505-1.133) \\
(0.379-1.198)\end{array}$ & $\begin{array}{l}24.9 \\
22.1\end{array}$ & $\begin{array}{r}15.2 \\
9.0\end{array}$ \\
\hline Child-Pugh score & $\begin{array}{l}5 \\
6\end{array}$ & $\begin{array}{r}118(76) \\
32(21)\end{array}$ & & $\rightarrow$ & - & $\begin{array}{l}0.758 \\
0.875\end{array}$ & $\begin{array}{l}(0.517-1.109) \\
(0.414-1.846)\end{array}$ & $\begin{array}{l}24.1 \\
12.0\end{array}$ & $\begin{array}{l}15.2 \\
12.4\end{array}$ \\
\hline Prior TACE & $\begin{array}{l}\text { None } \\
1-2\end{array}$ & $\begin{array}{l}93(60) \\
63(40)\end{array}$ & & - & & $\begin{array}{l}0.863 \\
0.474\end{array}$ & $\begin{array}{l}(0.561-1.328) \\
(0.276-0.812)\end{array}$ & $\begin{array}{l}22.5 \\
25.2\end{array}$ & $\begin{array}{l}15.2 \\
10.9\end{array}$ \\
\hline & & 0.001 & 0.010 & 0.100 & 1.000 & 10.000 & & & \\
\hline & & & $\begin{array}{l}\text { ith Sor } \\
\text { m bett }\end{array}$ & & $\begin{array}{l}\text { TACE } \\
\text { arm b }\end{array}$ & & & & \\
\hline
\end{tabular}

Fig. 6. Forest plot of updated TACE-specific PFS in subgroup analysis as of the data cut-off date of July $31,2020$.

to 47 patients (58.8\%) in the TACE plus sorafenib group $(p=0.014)$. The most common treatment after discontinuation of protocol treatment was transarterial therapy including hepatic arterial infusion chemotherapy. The reason for this is because during the most of the study period (2011-2020), only sorafenib was available until the time when other effective targeted agents are available. Therefore, mild TACE or hepatic arterial infusion chemotherapy was probably performed even after progression. In the TACE alone group, 29 patients (50.0\%) were administered sorafenib, and other post-trial anticancer treatments included regorafenib, lenvatinib, ramucirumab, or immunotherapy (Table 2).

\section{Patterns of unTACEable Progression}

Patterns of unTACEable progression included 68 patients $(43.6 \%)$ with a $25 \%$ or greater increase in intrahepatic lesions from the baseline tumor burden (sum of two-dimensional measurements $\leq 5$ intrahepatic lesions), 21 patients $(13.5 \%)$ with vascular invasion, 18 patients (11.5\%) with $\geq 10 \mathrm{~mm}$ extrahepatic metastasis lesions, 6 patients $(3.8 \%)$ with a transient Child-Pugh score $\geq 10$ points, and 8 patients (5.1\%) who fulfilled the definition of refractory to TACE. Additionally, 13 patients (8.3\%) died without unTACEable progression, 12 (7.7\%) had no events, and $10(6.4 \%)$ switched to other treatments before progression (online suppl. Table 4). There were no significant differences between the two arms in terms of the frequency of these events. 


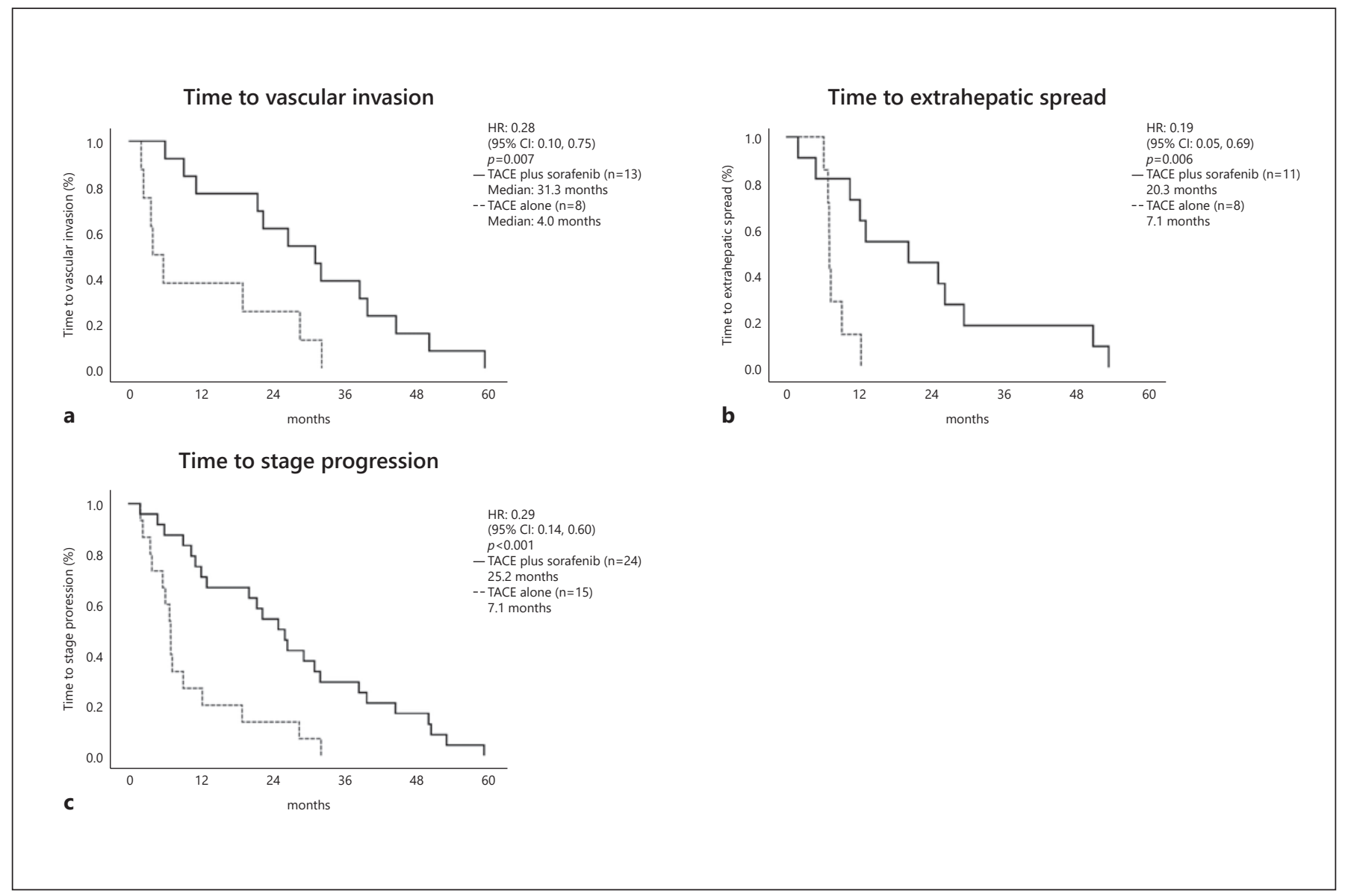

Fig. 7. a Time to MVI. b Time to EHS. c Time to stage progression.

Table 2. Anticancer treatment during survival follow-up

\begin{tabular}{|c|c|c|c|}
\hline Liver transplantation & $0(0.0)$ & $0(0.0)$ & \\
\hline Resection & $1(2.1)$ & $1(1.7)$ & \\
\hline \multicolumn{4}{|l|}{ Anticancer medication, $n(\%)$} \\
\hline Sorafenib & $5(10.6)$ & $29(50.0)$ & \\
\hline Regorafenib & $7(14.9)$ & $5(8.6)$ & \\
\hline Lenvatinib & $6(12.8)$ & $3(5.2)$ & \\
\hline Ramucirumab & $2(4.3)$ & $3(5.2)$ & \\
\hline Clinical trials (immunotherapy) & $4(8.5)$ & $5(8.6)$ & \\
\hline
\end{tabular}

Some treatment is overlapped. * Fisher's exact test. 'This therapy includes drug-eluting bead-TACE or hepatic arterial infusion chemotherapy. 
Table 3. All-grade treatment-emergent AEs within 8 weeks after first TACE and those with a frequency $\geq 10 \%$ in either group corresponding to grades 3 and 4

\begin{tabular}{|c|c|c|c|c|c|c|c|c|}
\hline Adverse event, $n(\%)$ & \multicolumn{4}{|c|}{ TACE plus sorafenib $(n=77)$} & \multicolumn{4}{|c|}{ TACE alone $(n=71)$} \\
\hline Elevated ALT & 69 (89.6) & $50(64.9)$ & $18(23.4)$ & $1(1.3)$ & $55(77.5)$ & $42(59.2)$ & $13(18.3)$ & $0(0.0)$ \\
\hline Thrombocytopenia & $67(87.0)$ & $57(74.0)$ & $10(13.0)$ & $0(0.0)$ & $53(74.6)$ & $51(71.8)$ & $2(2.8)$ & $0(0.0)$ \\
\hline Elevated bilirubin & $55(71.4)$ & $54(70.1)$ & $1(1.3)$ & $0(0.0)$ & $39(54.9)$ & $37(52.1)$ & $2(2.8)$ & $0(0.0)$ \\
\hline Hypertension & $45(58.4)$ & $37(48.1)$ & $8(10.4)$ & $0(0.0)$ & $28(39.4)$ & $25(35.2)$ & $3(4.2)$ & $0(0.0)$ \\
\hline Elevated lipase & $38(49.4)$ & $26(33.8)$ & $11(14.3)$ & $1(1.3)$ & $18(25.4)$ & $16(22.5)$ & $2(2.8)$ & $0(0.0)$ \\
\hline Elevated serum amylase & $32(41.6)$ & $26(33.8)$ & $6(7.8)$ & $0(0.0)$ & $19(26.8)$ & $18(25.4)$ & $1(1.4)$ & $0(0.0)$ \\
\hline Neutropenia & $30(39.0)$ & $26(33.8)$ & $4(5.2)$ & $0(0.0)$ & $29(40.8)$ & $29(40.8)$ & $0(0.0)$ & $0(0.0)$ \\
\hline Decreased WBC count & $29(37.7)$ & $28(36.4)$ & $1(1.3)$ & $0(0.0)$ & $26(36.6)$ & $26(36.6)$ & $0(0.0)$ & $0(0.0)$ \\
\hline Malaise & $20(26.0)$ & $20(26.0)$ & $0(0.0)$ & $0(0.0)$ & $9(12.7)$ & $9(12.7)$ & $0(0.0)$ & $0(0.0)$ \\
\hline Erythema multiforme & $9(11.7)$ & $7(9.1)$ & $2(2.6)$ & $0(0.0)$ & $0(0.0)$ & $0(0.0)$ & $0(0.0)$ & $0(0.0)$ \\
\hline Weight loss & $10(13.0)$ & $10(13.0)$ & $0(0.0)$ & $0(0.0)$ & $2(2.8)$ & $2(2.8)$ & $0(0.0)$ & $0(0.0)$ \\
\hline Hoarseness & $10(13.0)$ & $10(13.0)$ & $0(0.0)$ & $0(0.0)$ & $0(0.0)$ & $0(0.0)$ & $0(0.0)$ & $0(0.0)$ \\
\hline
\end{tabular}

ALT, alanine transaminase; AST, aspartate transaminase; WBC, white blood cell.

In patients who developed MVI or EHS, the time to MVI (31.3 vs. 4.0 months; $\mathrm{HR}=0.28$; $95 \% \mathrm{CI}, 0.10-0.75$; $p=0.007)$ and time to EHS (20.3 vs. 7.1 months; HR = 0.19 ; $95 \%$ CI, $0.05-0.69$; $p=0.006$ ) were significantly longer in the TACE plus sorafenib group than in the TACE alone group. Time to stage progression was also significantly longer in the TACE plus sorafenib group than in the TACE alone group (20.3 vs. 7.1 months; $\mathrm{HR}=0.19$; 95\% CI, 0.05-0.69; $p=0.006$ ) (Fig. 7).

\section{Safety Outcomes}

Table 3 shows the treatment-related AEs affecting more than $10 \%$ of either treatment group and Grades three and four AEs occurring within 8 weeks after the first TACE. Patients in the TACE plus sorafenib group experienced more AEs of all grades than patients in the TACE alone group, including hand-foot skin reaction $(66.2 \%$ vs. $0.0 \%$ ), hypertension ( $58.4 \%$ vs. $39.4 \%$ ), elevated lipase ( $49.4 \%$ vs. $25.4 \%)$, fatigue $(26.0 \%$ vs. $9.9 \%)$, diarrhea ( $16.9 \%$ vs. $0.0 \%)$, erythema multiforme ( $11.7 \%$ vs. $0.0 \%)$, weight loss (13.0\% vs. $2.8 \%)$, and hoarseness (13.0\% vs. $0.0 \%)$.

\section{Discussion}

The TACTICS trial was a randomized, open-label, multicenter trial comparing the safety and efficacy of the combination of TACE plus sorafenib with those of TACE alone in patients with unresectable HCC who were not eligible for resection or ablation. In this trial, TACE-specific PFS was significantly better in the TACE plus sorafenib group than in the TACE alone group. However, OS was not significantly prolonged, although a clinically meaningful benefit was observed ( $\triangle \mathrm{OS}=5.4$ months).

These results show that because tumor conditions are better in intermediate-stage HCC with no vascular invasion and no EHS than in advanced HCC, it is easier for patients to undergo post-trial treatments even after completing the protocol treatment. As such, patients in both groups, particularly those in the control group, showed a favorable prolongation of OS. The ratio of subsequent therapy in the control group of the present TACTICS trial was significantly higher at $76.3 \%$ than that in the control group of the recent Checkmate 459 trial (54\%), a clinical trial of first-line treatment for advanced-stage HCC [23], demonstrating that subsequent therapy after progression can be performed more frequently in patients 
with intermediate-stage HCC than in those with advanced HCC [24]. As described by Llovet et al. [24] in their AASLD consensus paper, "the realization that more than $60 \%$ of patients progressing after TACE receive effective next-line therapies may compel the adoption of PFS as an acceptable primary endpoint for major trials." As $76.3 \%$ of patients (sorafenib 50\%) in the control arm of the present TACTICS trial were administered subsequent therapy after protocol treatment, whereas there was almost no available effective therapeutic option in the TACE plus sorafenib group in the trial period (20112020), it would be difficult to demonstrate the OS benefit in patients with intermediate-stage HCC under the present status of HCC treatment, in which several active agents can be used in subsequent lines of treatment.

Although the TACTICS trial improved the OS rate in the TACE plus sorafenib group during the first 48 months, the prolongation of post-progression survival (PPS) due to post-trial treatments diluted the OS benefit after 48 months. A longer PPS results in greater dilution of the HR of OS even if the PFS per RECIST achieve a good HR [25]. The median PPS in the TACE alone and TACE plus sorafenib groups was 17.3 and 13.4 months, respectively, suggesting that effective subsequent therapy was a major factor contributing to the lack of a significant OS benefit in this trial.

Terashima et al. [25] analyzed the results of 56 studies and showed that the correlation between PPS and OS is greater than that between PFS and OS, and that in systemic therapy for advanced-stage HCC, OS is strongly influenced by PPS. Similarly, Llovet et al. [26] reported a good correlation between the HRs of PFS and OS based on previous clinical trials of advanced HCC (correlation coefficient of 0.84), suggesting that if the HR of PFS is $\leq$ 0.6 , the HR of OS is $\leq 0.8$, further indicating positive results for the OS endpoint; thus, an HR of PFS $<0.6$ may be a surrogate endpoint of OS in advanced HCC. However, similar examination of the relationship between the PFS HR and that of OS from the five previous TACE combination trials plus the current TACTICS trial on intermediate-stage HCC in a similar manner revealed a correlation coefficient of 0.56 , which is worse than that in advanced HCC; additionally, the regression line based on the weighted Pearson coefficient model was more gentle than that of advanced-stage HCC [26], suggesting that OS is strongly affected by PPS (online suppl. Fig. 1). Therefore, as the OS HR was 0.861 , although the TACE-specific PFS HR in the primary analysis of the TACTICS trial was $0.59(<0.6)$, with intermediate-stage HCC (as an earlier stage and better condition than advanced HCC), it was not possible to demonstrate the OS benefits under the current status of HCC treatment when several effective post-trial treatments became available [26]. Additionally, Celsa et al. [27] clearly stated that PFS per RECIST cannot be a surrogate endpoint for OS.

For cases with a high tumor burden, such as those outside of the up-to-seven criteria, the current consensus includes systemic therapy followed by selective TACE [10, $11,24,28,29$ ], especially in Asian countries. Prespecified analysis in this TACTICS trial showed that the median PFS of 22.1 months in the TACE plus sorafenib group was better than that of 9.0 months ( $\triangle \mathrm{PFS} 13.1$ months) in the patients outside of the up-to-seven criteria. Furthermore, in these patients, the median OS in the TACE plus sorafenib arm was 11.3 months longer than that in the TACE monotherapy arm (Fig. 4). This is consistent with previous results of sequential treatments using lenvatinib and TACE, and supports the concept of upfront systemic therapy followed by selective TACE treatments in patients with a high tumor burden $[10,11,28,30]$. Even among patients within the up-to-seven criteria, the median TACE-specific PFS in the TACE plus sorafenib group was 9.7 months longer than that in the TACE monotherapy group, whereas the median OS of 35.6 months was 3.7 months longer than the 31.9 months in the TACE monotherapy group (Fig. 4). These results suggest that preceding treatments with molecular-targeted drugs with anti-VEGF effects followed by selective TACE are effective for improving PFS and OS [10, 11, 28-30], especially for patients outside the up-to-seven criteria.

The TACTICS trial demonstrated no significant OS prolongation in the TACE plus sorafenib group relative to the TACE alone group. However, a clinically meaningful OS prolongation ( $\triangle \mathrm{OS}=5.4$ months) was achieved. Additionally, the TACE-specific endpoint is a valid endpoint that reflects the actual clinical progression from TACE and is, to some extent, a surrogate endpoint for OS since at least the OS rate up to 48 months was better in the combination arm. Furthermore, it is likely that this study did not show an OS benefit because all the patients had intermediate-stage HCC, resulting in a long PPS (13.4-17.3 months), and more patients received effective post-trial treatments than in studies involving subjects with advanced-stage HCC.

This study had a few limitations. First, we used an open-label design; therefore, post-study treatments may be influenced by investigator decisions. Second, this study was conducted as a phase II trial, and therefore may be underpowered to test the OS benefits. Third, relatively small number of patients was included in this trial. Exter- 
nal validation studies with a large number of patients are needed to confirm the results of this study. Fourth, TACEspecific PFS might not be clearly objective; therefore, the potential bias might not be excluded. Fifth, imaging review was done by investigators, not independent imaging review. This might be another potential bias.

In summary, although the TACTICS trial did not demonstrate a significant survival benefit of TACE plus sorafenib over TACE alone, it provides several important implications. First, combination treatments with molecular-targeted agents exerting an anti-VEGF effect significantly prolonged TACE-specific PFS. Second, TACE plus sorafenib showed a clinically meaningful and numerical prolongation of OS compared with TACE alone. Third, sequential treatment with a molecular-targeted agent with anti-VEGF effects and TACE contributed to prolonging the PFS and OS of patients with a tumor burden beyond the up-to-seven criteria. Finally, we report the longest OS observed in a clinical trial thus far $(36.2$ months) (online suppl. Table 5).

In conclusion, TACE plus sorafenib did not show statistically significant OS improvement. However, TACE plus sorafenib or systemic agent-TACE sequential therapy could be a treatment choice for intermediate-stage HCC since TACE-specific PFS was significantly improved. Additionally, TACE plus sorafenib/systemic therapy may be recommended for patients with a tumor burden beyond the up-to-seven criteria, since clinically meaningful OS prolongation with significantly improved TACE-specific PFS was observed in this trial.

\section{Statement of Ethics}

The protocol of this study was approved by the each institutional Ethics Committee or institutional review board in the each participated institution. The study was conducted in accordance with the 1964 Helsinki declaration and its later amendments or comparable ethical standards. Written informed consent was obtained from all patients.

\section{Conflict of Interest Statement}

Kudo M.: Lecture: Eisai, Bayer, MSD, BMS, EA Pharma, Eli Lilly, Chugai; Grants: Eisai, Takeda, Otsuka, Taiho, EA Pharma, Gilead Sciences, Abbvie, Sumitomo Dainippon Pharma, Chugai, Ono Pharma; Advisory Consulting: Eisai, Ono, MSD, BMS, Roche. Masatoshi Kudo is Editor-in-Chief of Liver Cancer. Ueshima K.: Honoraria from Bayer AG, Eisai Co. Ltd., Merck Sharp \& Dohme, Eli Lilly and Company, Chugai Pharmaceutical Co., Ltd., Takeda Pharmaceutical Co., Ltd., Pfizer Inc., Otsuka Pharmaceutical Co., Ltd., Sumitomo Dainippon Pharma Co., Ltd., Taiho Pharmaceuti- cal. Co. Ltd., EA Pharma Co., Ltd., and Kowa Co., Ltd.; Consulting or advisory fees from Eisai Co. Ltd., Eli Lilly and Company, Chugai Pharmaceutical Co., Ltd., Takeda Pharmaceutical Co., Ltd., and Pfizer Inc. Kazuomi Ueshima is an Editorial Board Member of Liver Cancer. Ikeda M. reports grants and personal fees from Bayer, grants and personal fees from Eisai, grants and personal fees from Eli Lilly Japan, grants and personal fees from Chugai Pharmaceutical, grants and personal fees from AstraZeneca, grants and personal fees from Merck Sharp \& Dohme, grants from Novartis, grants from Bristol Myers Squibb, grants from Merck Serono, personal fees from Sumitomo Dainippon Pharma, grants and personal fees from Takeda, outside the submitted work. Masafumi Ikeda is an Editorial Board Member of Liver Cancer. Aikata H.: Lecture: Eisai, Bayer. Izumi N.: Receiver speaker bureau from Bayer, Eisai, Lily, and Chugai. Namiki Izumi is an Associate Editor Member of Liver Cancer. Kuzuya T.: Lecture: Eisai, Bayer, Eli Lilly; Grants: Eisai. Isoda N.: Grants: GK, Eisai. Moriguchi M.: Honoraria: Eisai, Bayer, MSD, Eli Lilly, Chugai; Advisory Consulting: Bayer, Eli Lilly, Chugai. Ogasawara S.: Lecture: Eisai, Bayer, MDS, EA Pharma, Eli Lilly, Chugai, Takeda; Grants: Eisai, Bayer, Eli Lilly, AstraZeneca, Chugai; Advisory Consulting: Eisai, Chugai, Roche, AstraZeneca. Yoshimura K.: Lecture: AstraZeneca, Chugai Pharmaceutical Co., Ltd., Eizai, Otsuka, Nihon Kayaku, Elli Lilly, Taiho, Novartis, Boehringer Ingelheim, BrightPath Biotherapeutics, and Nihon Shinyaku, outside the submitted work. Okusaka T.: Consulting/advisory role: Taiho Pharmaceutical, Daiichi Sankyo, Sumitomo Dainippon Pharma, Bristol Myers Squibb, AstraZeneca, Eisai, and Nihon Servier; Research funding: Eli Lilly Japan, MSD, AstraZeneca, Eisai, Chugai Pharmaceutical, Novartis Pharma, Sumitomo Dainippon Pharma, Bristol Myers Squibb, Taiho Pharmaceutical, and Baxter; Honoraria: Meiji Seika Pharma, Teijin Pharma, Eli Lilly Japan, MSD, AstraZeneca, AbbVie, Eisai, Ono Pharmaceutical, Yakult Honsha, Daiichi Sankyo, Chugai Pharmaceutical, Nihon Servier, and Novartis Pharma; Scientific advisory board: AstraZeneca, Nippon Shinyaku, Nihon Servier, Novartis Pharma, Pfizer, and Mundipharma. Junji Furuse is an Editorial Board Member of Liver Cancer. Norihiro Kokudo is an Associate Editor Member of Liver Cancer.

\section{Funding Sources}

This work was supported by the Japan Liver Oncology Group with funding from Bayer Yakuhin Ltd., Japan under a research contract.

\section{Author Contributions}

Masatoshi Kudo and Kazuomi Ueshima contributed to the study design, data collection, analysis, manuscript writing, critical review of the manuscript, and final approval of the submitted manuscript. Masafumi Ikeda, Takuji Torimura, Nobukazu Tanabe, Hiroshi Aikata, Namiki Izumi, Takahiro Yamasaki, Shunsuke Nojiri, Keisuke Hino, Hidetaka Tsumura, Teiji Kuzuya, Norio. Isoda, Michihisa Moriguchi, Hajime Aino, Akio Ido, Naoto Kawabe, Kazuhiko Nakao, Yoshiyuki Wada, Sadahisa Ogasawara, Takuji Okusaka, Junji Furuse, Norihiro Kokudo, Kiwamu Okita, and Yasuaki Arai contributed to data collection, critical review of the manu- 
script, and final approval of the submitted manuscript. Philip Johnson contributed to interpretation of the data, critical review of the manuscript, and final approval of the submitted manuscript. Kenichi Yoshimura performed the statistical analysis, critical review of the manuscript, and final approval of the manuscript.

\section{Data Availability Statement}

All data relevant to the study are included in the article or uploaded as online supplementary material.

\section{References}

1 Llovet JM, Kelley RK, Villanueva A, Singal AG, Pikarsky E, Roayaie S, et al. Hepatocellular carcinoma. Nat Rev Dis Primers. 2021;7: 6.

2 Llovet JM, Bruix J. Systematic review of randomized trials for unresectable hepatocellular carcinoma: chemoembolization improves survival. Hepatology. 2003;37:429-42.

3 European Association for the Study of the Liver. EASL Clinical Practice Guidelines: management of hepatocellular carcinoma. J Hepatol. 2018;69:182-236.

4 Marrero JA, Kulik LM, Sirlin CB, Zhu AX, Finn RS, Abecassis MM, et al. Diagnosis, staging, and management of hepatocellular carcinoma: 2018 practice guidance by the American Association for the Study of liver diseases. Hepatology. 2018;68:723-50.

5 Lencioni R, de Baere T, Soulen MC, Rilling WS, Geschwind JF. Lipiodol transarterial chemoembolization for hepatocellular carcinoma: a systematic review of efficacy and safety data. Hepatology. 2016;64:106-16.

6 Li X, Feng GS, Zheng CS, Zhuo CK, Liu X. Expression of plasma vascular endothelial growth factor in patients with hepatocellular carcinoma and effect of transcatheter arterial chemoembolization therapy on plasma vascular endothelial growth factor level. World J Gastroenterol. 2004;10:2878-82.

7 Wang B, Xu H, Gao ZQ, Ning HF, Sun YQ, Cao GW. Increased expression of vascular endothelial growth factor in hepatocellular carcinoma after transcatheter arterial chemoembolization. Acta Radiol. 2008;49:523-9.

8 Jain RK. Normalization of tumor vasculature: an emerging concept in antiangiogenic therapy. Science. 2005;307:58-62.

9 Jiang H, Meng Q, Tan H, Pan S, Sun B, Xu R, et al. Antiangiogenic therapy enhances the efficacy of transcatheter arterial embolization for hepatocellular carcinomas. Int J Cancer. 2007;121:416-24.

10 Kudo M, Han KH, Ye SL, Zhou J, Huang YH, Lin SM, et al. A changing paradigm for the treatment of intermediate-stage hepatocellular carcinoma: Asia-Pacific primary liver cancer expert consensus statements. Liver Cancer. 2020;9:245-60.

11 Kudo M. A new treatment option for intermediate-stage hepatocellular carcinoma with high tumor burden: initial lenvatinib therapy with subsequent selective TACE. Liver Cancer. 2019;8:299-311.
12 Kudo M, Imanaka K, Chida N, Nakachi K, Tak WY, Takayama T, et al. Phase III study of sorafenib after transarterial chemoembolisation in Japanese and Korean patients with unresectable hepatocellular carcinoma. Eur J Cancer. 2011;47:2117-27.

13 Lencioni R, Llovet JM, Han G, Tak WY, Yang J, Guglielmi A, et al. Sorafenib or placebo plus TACE with doxorubicin-eluting beads for intermediate stage HCC: the SPACE trial. J hepatol. 2016;64:1090-8.

14 Meyer T, Fox R, Ma YT, Ross PJ, James MW, Sturgess R, et al. Sorafenib in combination with transarterial chemoembolisation in patients with unresectable hepatocellular carcinoma (TACE 2): a randomised placebo-controlled, double-blind, phase 3 trial. Lancet Gastroenterol Hepatol. 2017;2:565-75.

15 Kudo M, Cheng AL, Park JW, Park JH, Liang PC, Hidaka H, et al. Orantinib versus placebo combined with transcatheter arterial chemoembolisation in patients with unresectable hepatocellular carcinoma (ORIENTAL): a randomised, double-blind, placebo-controlled, multicentre, phase 3 study. Lancet Gastroenterol Hepatol. 2018;3:37-46.

16 Kudo M, Han G, Finn RS, Poon RT, Blanc JF, Yan L, et al. Brivanib as adjuvant therapy to transarterial chemoembolization in patients with hepatocellular carcinoma: a randomized phase III trial. Hepatology. 2014;60:1697707.

17 Kudo M, Ueshima K, Ikeda M, Torimura T, Tanabe N, Aikata H, et al. Randomised, multicentre prospective trial of transarterial chemoembolisation (TACE) plus sorafenib as compared with TACE alone in patients with hepatocellular carcinoma: TACTICS trial. Gut. 2020;69:1492-501.

18 Kudo M, Matsui O, Izumi N, Kadoya M, Okusaka T, Miyayama S, et al. Transarterial chemoembolization failure/refractoriness: JSHLCSGJ criteria 2014 update. Oncology. 2014; 87(Suppl 1):22-31.

19 Dmitrienko A, Offen WW, Westfall PH. Gatekeeping strategies for clinical trials that do not require all primary effects to be significant. Stat Med. 2003;22:2387-400.

20 Kudo M, Kubo S, Takayasu K, Sakamoto M, Tanaka M, Ikai I, et al. Response evaluation criteria in cancer of the liver (RECICL) proposed by the Liver Cancer Study Group of Japan (2009 Revised Version). Hepatol Res. 2010;40:686-92.
21 Takayasu K, Arii S, Matsuo N, Yoshikawa M, Ryu M, Takasaki K, et al. Comparison of CT findings with resected specimens after chemoembolization with iodized oil for hepatocellular carcinoma. AJR. 2000;175:699-704.

22 Bolondi L, Burroughs A, Dufour JF, Galle PR, Mazzaferro V, Piscaglia F, et al. Heterogeneity of patients with intermediate (BCLC B) hepatocellular carcinoma: proposal for a subclassification to facilitate treatment decisions. Sem Liver Dis. 2012;32:348-59.

23 Yau T, ParkJW, Finn RS, Cheng AL, Mathurin P, Edeline J, et al. Nivolumab versus sorafenib in advanced hepatocellular carcinoma (CheckMate 459): a randomised, multicentre, open-label, phase 3 trial. Lancet Oncol. 2022; 23:77-90.

24 Llovet JM, Villanueva A, Marrero JA, Schwartz M, Meyer T, Galle PR, et al. Trial design and endpoints in hepatocellular carcinoma: AASLD Consensus Conference. Hepatology. 2021;Suppl 1:158-91.

25 Terashima T, Yamashita T, Takata N, Nakagawa $\mathrm{H}$, Toyama T, Arai K, et al. Post-progression survival and progression-free survival in patients with advanced hepatocellular carcinoma treated by sorafenib. Hepatol Res. 2016;46:650-6.

26 Llovet JM, Montal R, Villanueva A. Randomized trials and endpoints in advanced HCC: role of PFS as a surrogate of survival. J Hepatol. 2019;70:1262-77.

27 Celsa C, Cabibbo G, Enea M, Battaglia S, Rizzo GEM, Busacca A, et al. Are radiological endpoints surrogate outcomes of overall survival in hepatocellular carcinoma treated with transarterial chemoembolization? Liver Int. 2021;41:1105-16.

28 Kudo M, Ueshima K, Chan S, Minami T, Chishina $\mathrm{H}$, Aoki $\mathrm{T}$, et al. Lenvatinib as an initial treatment in patients with intermediate-stage hepatocellular carcinoma beyond up-to-seven criteria and child-pugh a liver function: a Proof-Of-Concept Study. Cancers. 2019;11:1084.

29 Kudo M, Kawamura Y, Hasegawa K, Tateishi R, Kariyama K, Shiina S, et al. Management of hepatocellular carcinoma in Japan: JSH consensus statements and recommendations 2021 update. Liver Cancer. 2021;10:181-223.

30 Kawamura Y, Kobayashi M, Shindoh J, Kobayashi Y, Okubo S, Tominaga L, et al. Lenvatinib-transarterial chemoembolization sequential therapy as an effective treatment at progression during lenvatinib therapy for advanced hepatocellular carcinoma. Liver Cancer. 2020;9:756-70. 